\title{
Civil Aircraft System Integration Research
}

\author{
Li Haomin \\ shanghai aircraft design and research institute, comac \\ shanghai,China
}

\begin{abstract}
This paper according as the experience of china new civil aircraft developed, introduce the concept and methods of civil aircraft systems integration. Top-down system developed process was described. The systems integration and comprehensive approach, such as requirements analysis, functional analysis \& allocation, safety assessment, system design synthesis and verification, was detailed described. This philosophy and methods have reference and inspiration effect for developing large complex systems.
\end{abstract}

Keyword-sRequirements analysis, Functional analysis/Allocate, System integration, Safety assessment, Design synthesis, Verification

\section{INTRODUCTION}

Civil aircraft as a highly complex large integration systems, which characterized meet customer requirements based on ensuring safety. Based on this characteristic, the development process of civil aircraft generally use a topdown process, first determine the requirements, then the functional define, then the aircraft design, then the system design, component design and produce further, and then successive components to system integration verification, functional and requirements verification, and finally delivered to the user, shown as Figure 1.

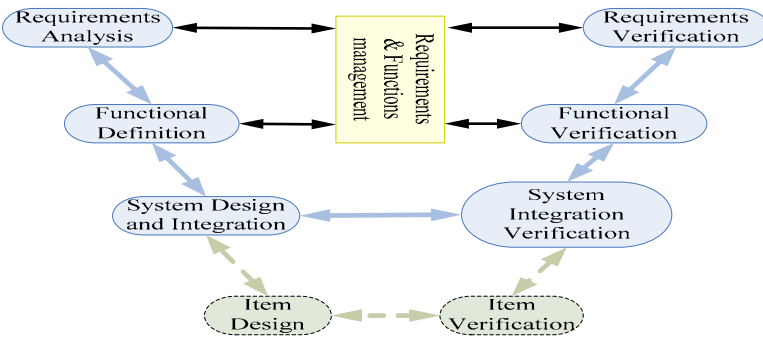

Figure 1. Civil aircraft develop process

The advantages of doing so is very obvious, to ensure that each requirements are met and the integrity of the design, to ensure compatibility between the systems, can be integrated, verification and airworthiness certification relatively easily, and better to understand the impact of a single function to the aircraft. International aircraft manufacturer has developed this top-down process applied to a number of new aircraft development process, and have solidified to the industry standard such as "ARP SAE 4754 guidelines to civil aircraft and systems development".

\author{
Wu Jianhua \\ shanghai aircraft design and research institute, comac \\ shanghai,China
}

\section{SYSTEM INTEGRATION PROCESS}

The Systems Integration Process is a comprehensive, iterative and recursive problem solving process, applied sequentially top-down by integrated teams. It transforms needs and requirements into a set of system product and process descriptions, generate information for decision makers, and provides input for the next level of development. The process is applied sequentially, one level at a time, adding additional detail and definition with each level of development. As shown by Figure 2, the process includes: inputs and outputs; requirements analysis; functional analysis and allocation; requirements loop; synthesis; design loop; verification; and system analysis and control[1].

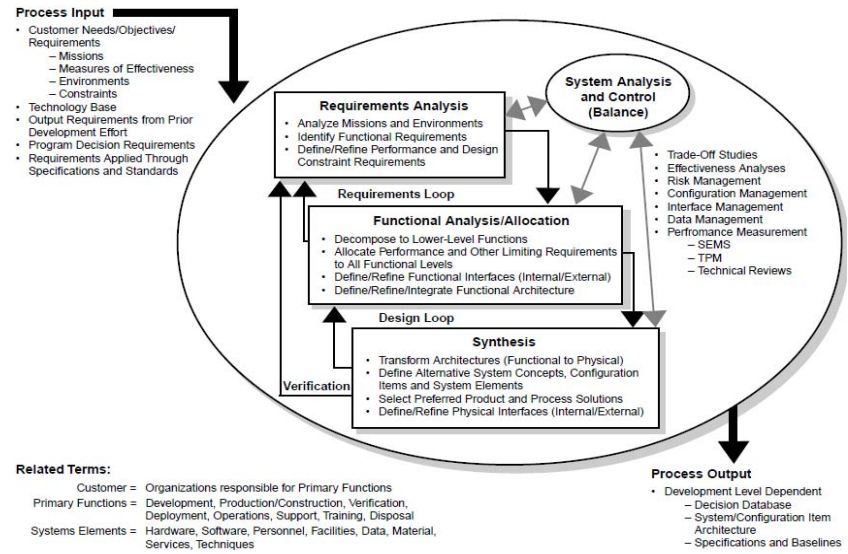

Figure 2. The system integration process

- System Integration Process Inputs: Input consist primarily of the customer's needs, objectives, requirements and project constraints.

- Requirements Analysis: Requirements analysis is used to develop functional and performance requirements; that is, customer requirements are translated into a set of requirements that define what the system must do and how well it must perform.

- Functional Analysis/Allocation: Functions are analyzed by decomposing higherlevel functions identified through requirements analysis into lower-level functions. The performance requirements associated with the higher level are allocated to lower functions.

- Safety Assessment: The safety assessment process is used by a company to show compliance with certification requirements and in meeting its own internal safety standards. [2]. 
- Design Synthesis is the process of defining the product or item in terms of the physical and software elements which together make up and define the item.

- Verification: For each application of the system engineering process, the solution will be compared to the requirements. This part of the process is called the verification loop, or more commonly, Verification. Each requirement at each level of development must be verifiable.

\section{REQUIREMENTA ANALYSIS}

\section{A. Type Of Requirements}

Requirements are categorized in several ways. The following are common categorizations of requirements that relate to technical management:

- Customer Requirements: Statements of fact and assumptions that define the expectations of the system in terms of mission objectives, environment, constraints, and measures of effectiveness and suitability.

- Functional Requirements: The necessary task, action or activity that must be accomplished. Functional (what has to be done) requirements identified in requirements analysis will be used as the toplevel functions for functional analysis.

- Performance Requirements: The extent to which a mission or function must be executed; generally measured in terms of quantity, quality, coverage, timeliness or readiness.

- Derived Requirements: Requirements that are implied or transformed from higher-level requirement. For example, a requirement for long range or high speed may result in a design requirement for low weight.

- Allocated Requirements: A requirement that is established by dividing or otherwise allocating a high-level requirement into multiple lower-level requirements.

\section{B. Requirements Analysis}

Requirements analysis involves defining customer needs and objectives in the context of planned customer use, environments, and identified aircraft characteristics to determine requirements for aircraft functions. Prior analyses are reviewed and updated, refining mission and environment definitions to support aircraft definition.

The purpose of Requirements Analysis is to:

- Refine customer objectives and requirements;

- Define initial performance objectives and refine them into requirements;

- Identify and define constraints that limit solutions; and

- Define functional and performance requirements based on customer provided measures of effectiveness.

\section{Requirements Analysis Output}

The requirements that result from requirements analysis are typically expressed from one of three perspectives or views. These have been described as the Operational, Functional, and Physical views.

- Operational View: The Operational View addresses how the system will serve its users.
- Functional View: The Functional View focuses on WHAT the system must do to produce the required operational behavior. It includes required inputs, outputs, states, and transformation rules.

- Physical View: The Physical View focuses on HOW the system is constructed. It is key to establishing the physical interfaces among operators and equipment, and technology requirements.

\section{IV. . FUNCTIONAL ANALYSIS AND ALLOCATION}

\section{A. Functional Analysis And Allocation}

The purpose of functional analysis and allocation is to transform the functional, performance, interface and other requirements that were identified through requirements analysis into a coherent description of system functions that can be used to guide the Design Synthesis activity that follows. The designer will need to know what the system must do, how well, and what constraints will limit design flexibility.

This top-down process of translating system level requirements into detailed functional and performance design criteria includes:

- Defining the system in functional terms, then decomposing the top-level functions into subfunctions,

- Translating higher-level performance requirements into detailed functional and performance design criteria or constraints,

- Identifying and defining all internal and external functional interfaces,

- Identifying functional groupings to minimize and control interfaces (functional partitioning),

- Determining the functional characteristics of existing or directed components in the system and incorporating them in the analysis and allocation,

- Performing trade studies to determine alternative functional approaches to meet requirements.

\section{B. Functional Architecture}

The functional architecture is a top-down decomposition of aircraft functional and performance requirements. The architecture will show not only the functions that have to be performed, but also the logical sequencing of the functions and performance requirements associated with the functions. It also includes the functional description of existing.

This is an example of functional analysis and architecture. Functions come mainly from requirements and aircraft operation philosophy. Modern civil aircraft operating philosophy is the safety of passengers and cargo movement from the beginning to the end, with minimal impact to the environment, and ensure the operational efficiency and continuing airworthiness, the aircraft must be maintained. In support of flight, must provide the human interface, hydraulic energy, gas supply and power supply and other public resources. So the aircraft basic function is payload, move, provide common resource, ensure safety, support operation and compatibility with environment, shown as Figure 3. 


\section{SAFETY ASSESSMENT}

Safety assessment is a important and complex aircraft system integration work. The output of safety assessmemt confirm many requirements of system and item design, and the safety assessment process can validate the system Figure 4 shows the fundamental relationships between these four specific assessments and the system development processes.

Safety assessment includes:

- Functional Hazard Assessment (FHA): Examines aircraft and system functions to identify potential functional failures and classifies the hazards associated with specific failure conditions.

- Preliminary Aircraft Safety Assessment/Preliminary System Safety Assessment (PASA/PSSA): Establish the aircraft or specific system or item safety requirements and provide a preliminary indication that the anticipated aircraft or system architectures can meet those safety requirements.

- Aircraft Safety Assessment/System Safety Assessment (ASA/SSA): Collects, analyzes, and documents verification that the aircraft and systems, as implemented, meet the safety requirements established by the PASA and the PSSA.

- Common Cause Analysis (CCA): Establishes and verifies physical and functional separation, isolation and independence requirements between systems and items and verifies that these requirements have been met.

\section{DESIGN SYNTHESIS}

Design synthesis is a creative activity that develops a physical architecture (a set of product, system, and/or software elements) capable of performing the required functions within the limits of the performance parameters prescribed. The objective of design synthesis is to combine and restructure hardware and software components in such a way as to achieve a design solution capable of satisfying the stated requirements. Design synthesis begins with the output of Functional Analysis and Allocation (the functional architecture). The functional architecture is transformed into a physical architecture by defining physical components needed to perform the functions identified in Functional Analysis and Allocation.

Depending on the nature of the system and the development process used, initial item electronic hardware and software synthesis may occur on breadboards, prototypes, computer emulation, lab or flight-worthy articles. The output is equipment under configuration control together with development assurance data and hardware and/or software life cycle data. Detailed procedures developed during the design-build process should be used to verify that all electronic hardware and software synthesis requirements are met.

\section{VERIFICATION}

The objectives of the Verification process include using established criteria to conduct verification of the physical architecture (including software and interfaces) from the lowest level up to the total system to ensure that cost, schedule, and performance requirements are satisfied with acceptable levels of risk.

System design solutions are verified by the following types of activities:

- Analysis - the use of mathematical modelingand analytical techniques to predict the compliance of a design to its requirements based on calculated data or data derived from lower level component or subsystem testing. It is generally used when a physical prototype or product is not available or not cost effective.

- Inspection - the visual examination of the system, component, or subsystem. It is generally used to verify physical design features or specific manufacturer identification,

- Demonstration - the use of system, subsystem, or component operation to show that a requirement can be achieved by the system. It is generally used for a basic confirmation of performance capability and is differentiated from testing by the lack of detailed data gathering, or

- Test - the use of system, subsystem, or component operation to obtain detailed data to verify performance or to provide sufficient information to verify performance through further analysis. Testing is the detailed quantifying method of verification, and as described later in this chapter, it is ultimately required in order to verify the system design..

\section{VIII. . SUMMARIES}

Large and complex system require added design discipline and development structure to ensure that safety and operational requirements can be fully realized and substantiated. A top-down sequence for developing a specific system implementation from knowledge of an intended aircraft function provides a convenient conceptual model for the system development process. A typical system development progresses in an iterative and concurrent fashion using both top-down and bottom-up strategies.

\section{REFERENCES}

[1] Systems Engineering Fundamentals. SYSTEMS MANAGEMENT COLLEGE. DEPARTMENT OF DEFENSE. January 2001

[2] SAE ARP4754A Guidelines for Development of Civil Aircraft and Systems. 12.2010 


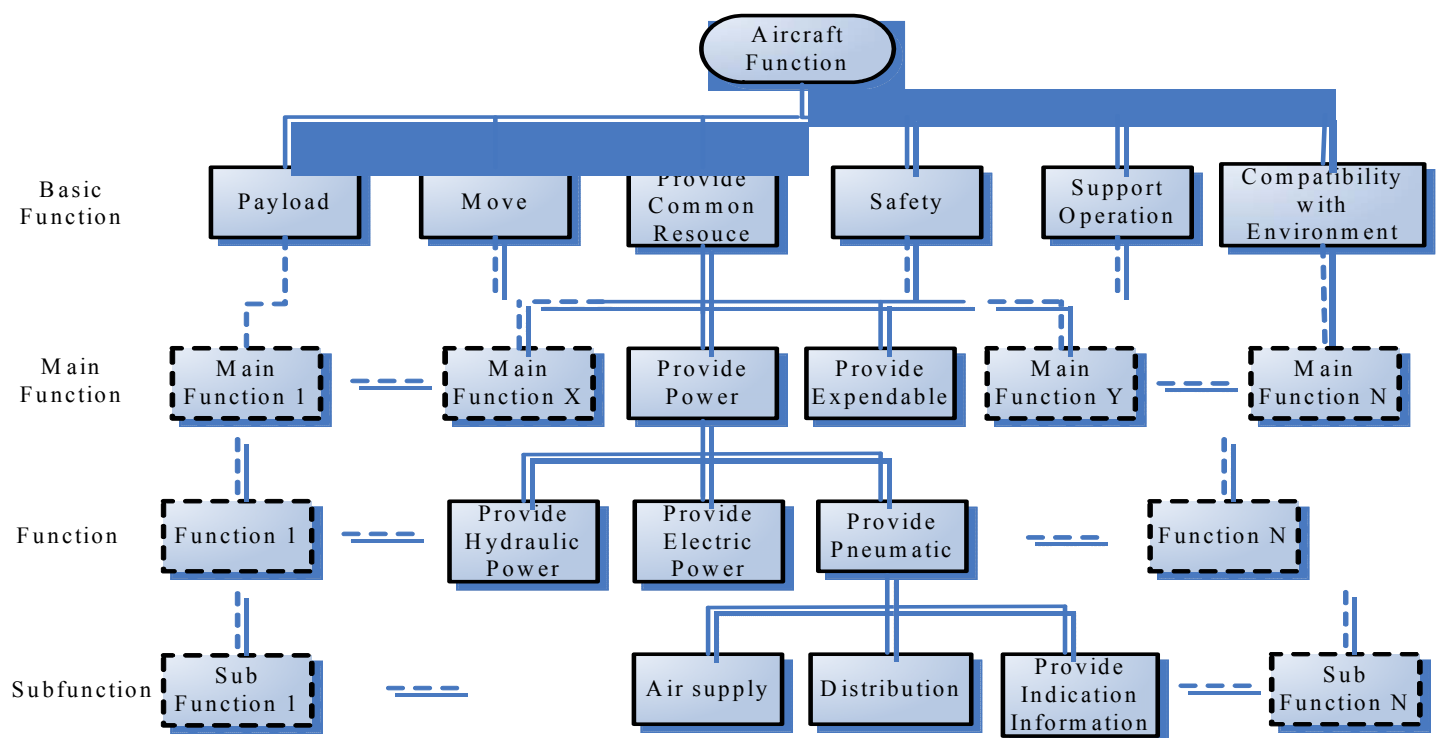

Figure 3. An example of arcraft fnctional achitecture

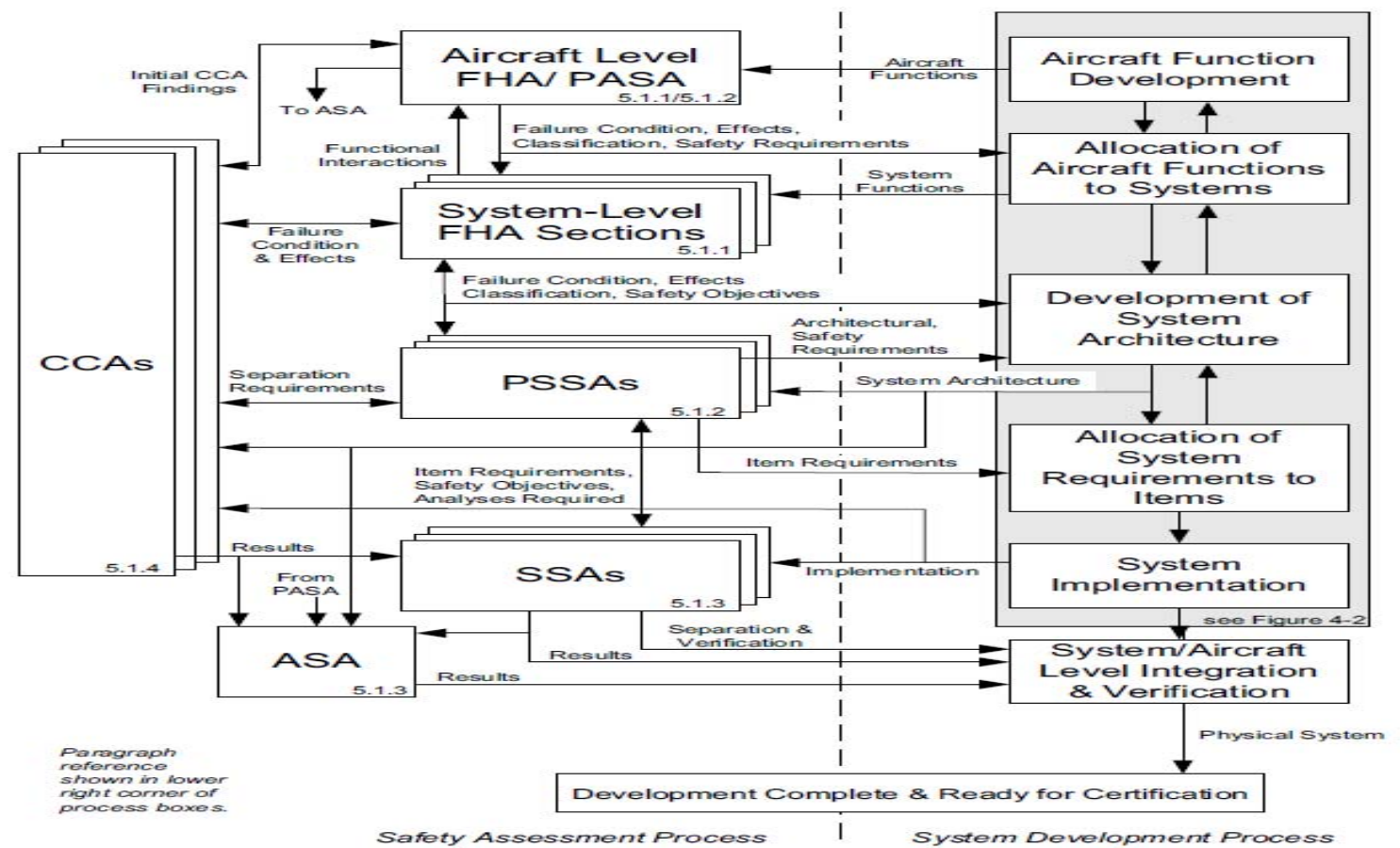

Figure 4. Safety assessment process mode[2] 